\title{
Complex 3D Shape Recovery using Hybrid Geometric Shape Features in a Hierarchical Shape Segmentation Approach
}

\author{
Hongwei Zheng and Dietmar Saupe \\ Department of Computer and Information Science \\ University of Konstanz, D-78457 Konstanz, Germany \\ \{Hongwei.zheng, Dietmar.Saupe\}@uni-konstanz.de
}

\begin{abstract}
We present a novel and reliable approach for complex object acquisition and surface registration using hybrid geometric shape features in a hierarchical 3D shape approximation and segmentation approach. First, instead of relying on one type of scanned data, we propose to use hybrid data provided that it can support both global and local geometric shape features. The scanned low-resolution global data supplies the global shape prior for registering the high-resolution local surface patches. Local surfaces can thus be optimally registered requiring less overlap and reducing uncertainty. Second, we cannot directly register huge volumes of data simultaneously due to the memory bottlenecks. We segment the global low-resolution model into several meaningful sub-shapes extending a hierarchical algorithm. The local surfaces can be registered on the sub-shapes respectively and all sub-shapes can be merged and rendered after registration. To verify the reliability of the approach, various $3 D$ models have been acquired. The experiments show compelling results by reconstructing very detailed models of complex objects. The approach can be applied to practical 3D modeling applications.
\end{abstract}

\section{Introduction}

3D shape acquisition and surface registration is important in computer vision, graphics, and related fields. Recent 3D acquisition techniques like structured light, coded light, time-of-flight, Moire interferometry, and triangulationbased laser range scanners have been gradually developed. However, efficient and high-quality 3D acquisition and surface registration is still a hard problem, especially for large and complex 3D shape models. Since most 3D acquisition and reconstruction systems can only reconstruct or acquire certain 3D patches, it is still difficult to achieve a complete high-quality 3D shape. Even for laser scanner 3D acquisition systems, it is still not easy to get a high-quality 3D

Konstanzer Online-Publikations-System (KOPS)

URN: http://nbn-resolving.de/urn:nbn:de:bsz:352-opus-108611

URL: http://kops.ub.uni-konstanz.de/volltexte/2010/10861 shape model using large number of scanned range images for complex real objects.

In practice, due to the large space of possible 3D shape deformations, transformations, and complexities of noises, registration of local surface patches tend to be locally ambiguous and globally incoherent. It is hard to select the most suitable scanned local surface patches from many scans and discard the other scans. Furthermore, it is difficult to find naturally global geometric shape features which can optimally to aid local surface registration. To register local surface patches without using global measure criteria, small alignment errors for local patches may accumulate to large distortion [17], [20]. Finally, to acquire a large and complex 3D model, we need to process large number of scanned surface patches. However, due to the main memory bottleneck, we cannot process all high-resolution local surface patches simultaneously. We need to register the surface sequentially without keeping all the scans simultaneously in memory.

To solve these difficulties, a considerable amount of research has been dedicated to 3D surface registration from different sides such as rigid pairwise local surface registration, rigid global surface registration and nonrigid surface registration. A classical method for rigid pairwise local surface registration is the Iterative Closest Points method (ICP) [3], [4]. A similar method named free-form surface registration with adaptive threshold is also independently proposed by [28]. Later on, a sampling based ICP method has been proposed [15] for improving local patch alignment using constraints of rigid transformation. Rigid global surface registration methods have been proposed for simultaneously registering all local scans in rigid transformation [8]. In this case, since some registration errors are easily accumulated through the alignment of local patches, other methods focus on reducing the errors, e.g., closed-form methods [2], parallelizable pairwise alignment [22], and so on.

Nonrigid surface registration methods can be considered as an extension of the Thin-Plate-Splines (TPS) [5] method, e.g., non-rigid-shape with rigid-component warping [16], [7], vertex affine transformation [1], extended TPS [9], and 


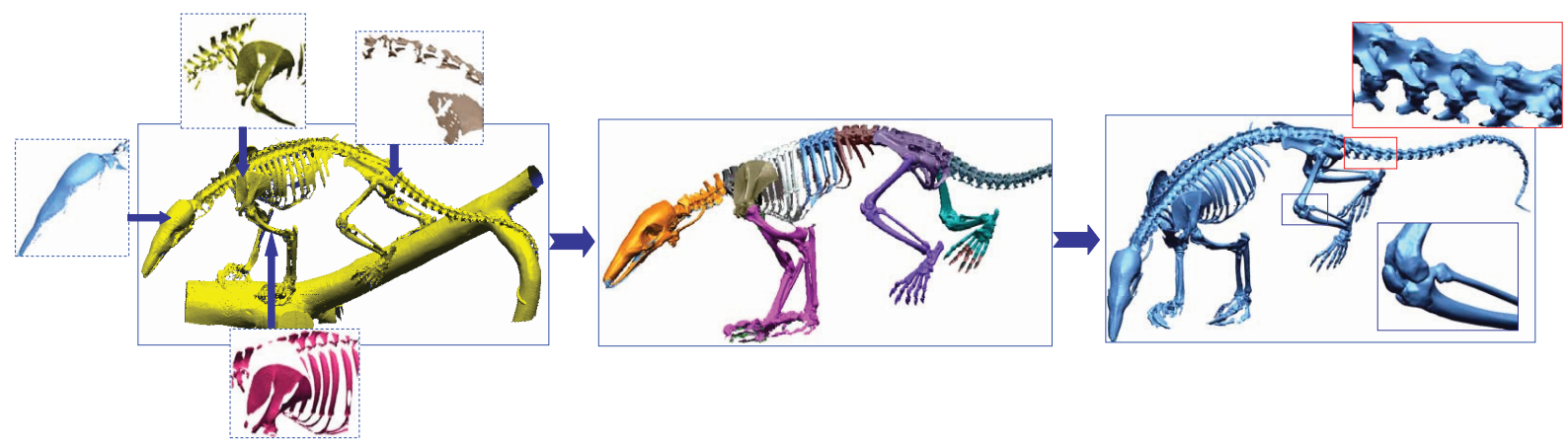

Figure 1. $a|b| c$. Complex 3D shape acquisition using local-global geometric shape features in a hierarchical shape segmentation algorithm. (a) Step 1: aligning high-resolution local surface patches to a low-resolution global shape. (b) Step 2: semantic-oriented segmentation to sub-shapes, and process the sub-shapes individually. (c) Step 3: merge the processed sub-shapes into a complete 3D shape.

surface interpenetration measure based genetic algorithm [23]. In practice, most 3D surface registration methods use certain assumptions. For example, the ICP algorithm needs a good initial alignment [3], [4]. Also, some measure criteria are also used for local feature extraction [14], [25] due to high-dimensional feature voting spaces. The global measure criteria [19] are used for unifying the registration solution.

A meaningful approximation and segmentation of 3D shapes can help us to avoid the memory bottlenecks for registering huge volumes of scanning data. We also need some semantic meaningful annotation for partitions so that we can understand the acquired data to fully exploit its potential. However, different segmentation methods may have different performances on such real complex 3D shapes. The main difficulty in region growing approaches is the choice of the seed points and of their number. Even if the number of regions is known, region growing approaches may be easily trapped in local minima, and heuristic methods are often required to escape from such situations. For example, given a complex 3D shape model, it is often very hard to segment the model into several meaningful parts.

The approach of hierarchical face clustering has been proposed by Garland and Heckbert [12], [13] and has been extended for variational 3D shape approximation [10]. Recently, Marco et al. [18] extend this approach for hierarchical 3D shape segmentation. The method produces high quality approximations in practice using differential geometry and approximation theory. The optimization of shape approximation is modeled in a discrete, variational geometric partitioning problem which is driven by minimizing the global approximation error. Inspired from these methods, we propose to segment the 3D shapes based on the combination of hierarchical 3D shape approximation and the model fitting measure of several primitive shapes.

In this paper, we propose a novel approach for acquiring large and complex 3D shape models combining highresolution local surface patches and a low-resolution global
3D model. A meaningful 3D shape approximation for a low-resolution global 3D model makes the computation feasible and reliable. Fig. 1 shows sketch of the approach. Our contribution is three-folds. (i) In contrast to most scanning approaches, we propose to use hybrid scanning data for 3D shape acquisition and surface registration, e.g., high-resolution local surface patches together with a lowresolution global shape structure model. In place of many small local patches, we can work with a global scan together with fewer local scans. The local scans are registered not only pairwise but also simultaneously with respect to the global shape model. (ii) The registration of local scans to a coarse global model prevents the accumulation of errors which is a common problem in sequential registration of multiple scans with slightly overlapping scans. (iii) We address the problem of memory bottlenecks so that the computation of the proposed approach is possible in practice. Large volumes of high-resolution local surface data can be computed sequentially and respectively using a global geometric feature prior. This approach is robust in the presence of noise and outliers. The advantages of the approach are demonstrated showing that high-accuracy can be obtained in registration of the high-resolution scans. Experiments show that the proposed approach outperforms previous ones. This approach can achieve high-quality surface registration of large and complex 3D shapes.

The paper is organized as follows. Sec. 2 shows rigid and nonrigid surface registration methods. Sec. 3 shows an extended hierarchical shape segmentation method for meaningful semantic segmentation. The integration of high-resolution local surfaces and the low-resolution global shape model are discussed in Sec. 4. Experimental results are shown in Sec. 5. Conclusions are summarized in Sec. 6.

\section{Rigid and Nonrigid Surface Registration}

In our project, two types of scanning data are acquired using a hybrid scanning system. A high-resolution stationary range scanner can achieve high-resolution scanning 


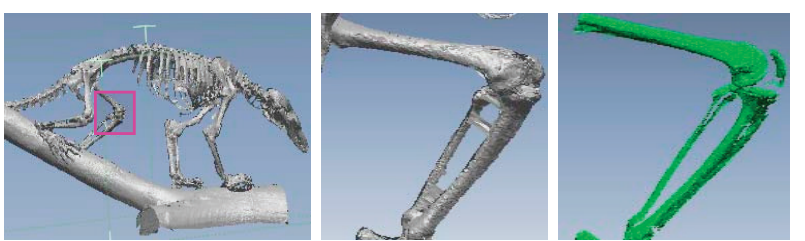

Figure 2. (a) A scanned low-resolution complete 3D shape model. (b) Zoom in (a). (c) A scanned high-resolution local surface patch.

patches of the 3D object, see Fig. 2 (c). We also use a light and movable hand-held laser scanner to acquire a complete low-resolution global shape model within a continuous scanning period around the object, see Fig. 2 (a)(b). Using the hybrid scanning data, our proposed approach needs to process not only pairwise rigid local surface alignment but also local-to-global non-rigid surface registration using the global geometric shape features, shown in Fig. 1. In the work diagram, firstly, high-resolution local surfaces are aligned onto the low-resolution global shape model using the rigid ICP method. In this way, we can still keep original fidelity of high-resolution local surfaces after the alignment. Secondly, due to memory bottleneck problem, we segment the low-resolution global shape model into several meaningful sub-shapes so that we process and refine the alignment sequentially and efficiently. Simultaneously, each aligned local surface is non-rigid adjusted and refined using a global nonrigid TPS bending algorithm based on segmented sub-shapes.

\subsection{Rigid Local Surface Registration}

According to the ICP algorithm, the rigid pairwise local surface registration algorithm can be described as follows. A 3D surface registration method seeks to find the best transformation $\mathcal{T}$ that relates two entities $P$ and $Q$ whose 3D surface are given by $R_{P}$ and $R_{Q}$, respectively. Thus, the goal of a registration algorithm is to find $\mathcal{T}$ such that the following objective function, $J\left(R_{P}, R_{Q}\right)$ is minimized,

$$
J\left(R_{P}, R_{Q}\right)=\sum_{P \in R_{P}}\left\|\mathcal{T}_{P}-\Psi(P)\right\|
$$

where $\Psi: P \rightarrow Q$; for $\forall P \in R_{P}, \Psi(P) \in R_{Q}$. The transformation $\mathcal{T}$ is used to optimally align entities $P$ and $Q$, and it usually depends upon the distortions which are presented on the surfaces in $3 \mathrm{D}$ space. Thus, $\mathcal{T}$ may be rigid, affine, polynomial, or elastic. In practice, the function $\Psi$ is usually unknown. We need to compute the $\Psi$ including translational and rotational part. The translational part of $\Psi$ brings the center of mass of $P$ to the center of mass of $Q$. The rotational part of $\Psi$ can be obtained as the unit eigenvector that corresponds to the maximum eigenvalue of a symmetric $4 \times 4$ matrix. The solution eigenvector is a unit quaternion description of the rotational part of $\Psi$. During the iterative computation, the position of the data points are updated via $P_{\text {cur. }}=\Psi\left(P_{\text {pre. }}\right)$. While the mean square error is smaller

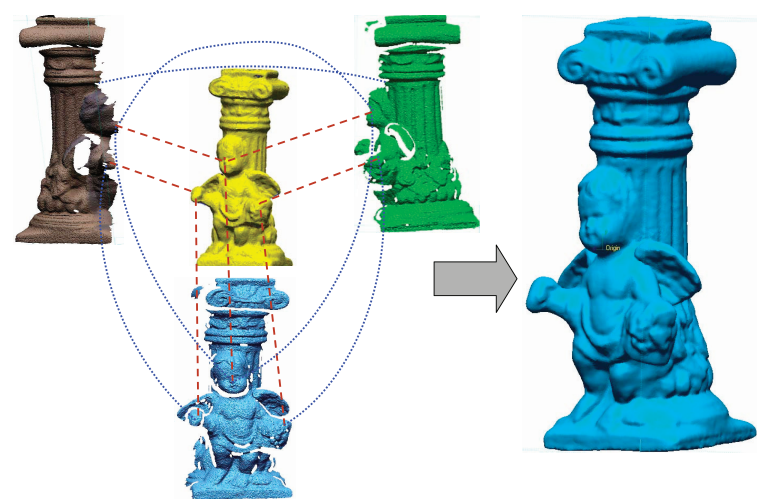

Figure 3. Pairwise surface correspondences (blue lines) and features in optimal global positions (dotted lines) via iterative localto-global nonrigid adjustment.

than a given threshold, the algorithm converges monotonically to a local minimum. When a good initial value is given, the algorithm can achieve a global convergence.

This algorithm is directly used in our experiments for rigid pairwise local-surface registration. For example, Fig. 3 shows pairwise local surface registration in blue line. However, as we have described before, small alignment errors may be accumulated to a gross error for the final alignment. Also, the additional non-rigid warp causes some serious problems, e.g., large deformation and distortion, shown in Fig. 9 (b)(c)(d). We need to simultaneously process local and global surface registration using hybrid scanning data. The diagram of our approach is shown in Fig. 3. In this diagram, pairwise local surface are aligned in the rigid ICP method so that we can keep more accuracy of those aligned local surface patches. Simultaneously, each local surface alignment error is diffused using a global nonrigid shape adjustment algorithm. This gives us a completely automatic local-to-global surface registration pipeline.

In practice, two fundamental questions of using the ICP algorithm need to be solved for surface registration. Similar to most non-linear minimization algorithms, the first question is how to construct an initial alignment for any given point sets to ensure a correct global convergence. The second question is how to construct an efficient mechanism that can adaptively align all scanned local patches to have an efficient registration with respect to the correct global minimum. Traditionally, it is hard to solve these two questions using a multitude of scanned local surfaces. In most cases, the initialization can be obtained by using knowledge about the position of the 3D sensors or by user input. If it is not possible, more complicated techniques like principal component analysis with a constrained exhaustive search [8] become necessary. Using hybrid scanning data, the complexity of searching a good initial alignment is simplified. The scanned low-resolution global shape model supplies accurate initial global feature points. 

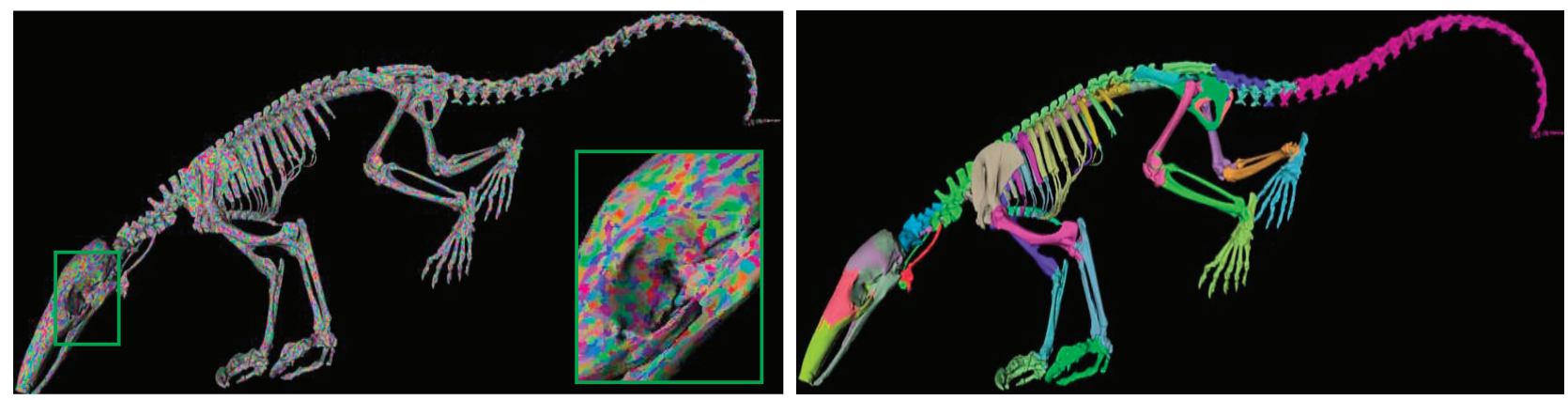

Figure 4. $a \mid b$. Hierarchical surface clustering and merging for shape approximation. (a). 178951 clusters. (b) 100 clusters.

\subsection{Non-rigid Global Surface Registration}

We use the thin-plate-spline (TPS) method for global registration by mapping each local feature point onto its global position. The TPS method [11] has been introduced to 3D geometric deformation processing [5]. The TPS has an algebra expressing the dependence of the physical bending energy of a thin plate on point constraints. For interpolating a surface over a fixed set of nodes in plane, the bending energy is a quadratic form in the heights assigned to the surface. On the other hand, the role of this bending function is similar to the approach of Laplacian-based mesh deformation [6].

The algorithm of TPS is a non-rigid, globally smooth function including affine and non-affine components. The non-affine warping component means that the sum of squares of all second order partial derivatives is minimized. Such functionality can be used for 3D mapping and it is easily computable. Thus, if $\xi: R^{n} \rightarrow R$ is an n-dimensional thin-plate spline, the bending energy,

$$
J_{\xi}=\int\left(\sum_{i, j} \xi_{p_{i} p_{j}}^{2}\right) d p_{1} \ldots d p_{n}
$$

is minimized. Given two corresponding point sets $P=$ $\left\{p_{1} \ldots p_{n}\right\}$ and $Q=\left\{q_{1} \ldots q_{n}\right\}$, there is a unique function $\xi$ such that $\xi\left(p_{i}\right)=q_{i}$ and their bending energy is minimal. Furthermore, this function takes the form $\xi(x)=$ $A x+B K(x)$ for summing affine component and non-affine warping component. Where $x$ is a point written in homogeneous coordinates, $A$ is an affine transformation matrix, $B$ is a fixed $n$-dimensional row vector of non-affine warping parameters constrained to $B P^{T}=0$, and $K(x)$ is an $n$-dimensional column vector where $K(x)_{i}$ is the Green's function $G\left|x-p_{i}\right|$. In other words, the TPS kernel contains the information about the point-sets' internal structural relationships and generates a non-rigid warp when combined with the warping coefficient matrix $B$. Thin-plate splines need not be interpolating. Instead, it can be formulated in a regularization energy functional according to [11].

$$
J_{\lambda}=\frac{1}{n} \sum_{i=1}^{n}\left\|q_{i}-\xi\left(p_{i}\right)\right\|^{2}+\lambda J_{\xi}
$$

This equation means that the TPS fits a mapping function $\xi\left(p_{i}\right)$ between corresponding point-sets $P$ and $Q$ by minimizing the energy. The first term is a distance measure term between two point sets. Since $\xi\left(p_{i}\right)$ is not unique, we need a smoothness constraints to regulate the mapping [11].

Here, the spline will not be interpolated, but for any fixed $\lambda$, there is a unique minimum. The warping strength depends on the regularization parameter $\lambda$. While $\lambda$ is close to zero, we get exact alignment of corresponding surface vertices. If $\lambda$ is zero, interpolation is exact and as it approaches infinity, the resulting TPS surface is reduced to a least squares fitted plane, i.e., "bending energy" of a plane is 0 . For the interpolating case, the thin-plate spline specification provides a linear system of equations.

To apply this equation to solve 3D point sets, based on [11], [27], we perform a QR factorization on $\Psi$ using the approximated equation ,

$$
A P+B(K+n \lambda I)=G
$$

with the condition of $B P^{T}=0$. Where $\mathrm{K}$ is $n \times n$ and $K_{i j}=G\left(\left|p_{i}-p_{j}\right|\right)$ which is a Green's function. We set a small value of $\lambda$ to improve the stability of numerical computation for this functional. In contrast to some previous work [22], [7], [17], the alignment of numerous local surfaces are globally controlled by the low-resolution global shape model. Since we need to have precise alignments for rigid local scans using the feature prior from the lowresolution global shape model, the spline must be heavily weighted toward interpolation for connecting and aligning slightly-overlapped high-resolution local surface patches. For this reason we can rely on accurate correspondences to produce a good local surface alignment without global shape distortion or error.

\section{Hierarchical 3D Shape Approximation}

This step addresses memory bottlenecks as it does not require keeping all the scans simultaneously in the main memory in computers. In Fig. 1, the total size of data is $1.162 \mathrm{~GB}$ and we need to process the data using a divide-and-conquer strategy. Therefore, a hierarchical 3D shape approxima- 
tion and clustering method is extended to segment the lowresolution global shape model into several meaningful parts for registering high-resolution local-surface patches.

Following the recent work of hierarchical face clustering methods on 3D meshes [13], [18], we define a set $\Theta$ as a solution space of geometric primitive models which consists of primary parametric geometric models as $\Theta=$ $\left\{M_{j}(\theta), j=1,2,3, \ldots, N\right\} . M_{j}(\theta)$ represents the $j$ th geometric model with its own parameters $\theta$, and $N$ is the number of primitive models.

$M_{j}(\theta)=\left\{\begin{array}{l}M_{1}\left(x_{i}, n_{i}\right), \text { planes } \\ M_{2}\left(c_{i}, r_{i}\right), \\ c_{i} \text { is the sphere center, } r_{i} \text { is radius } \\ M_{3}\left(x_{i}, d_{i}, r_{i}\right), \\ x_{i} \text { a point on the axis, } d_{i} \text { direction, } r_{i} \text { radius }\end{array}\right.$

$M_{1}$ is a plane with a point $x_{i}$ and a normal $n_{i}, i$ is the index of point. $M_{2}$ is a sphere and can be characterized its center $c_{i}$ and a radius $r_{i} . M_{3}$ is a cylinder with a point $x_{i}$ on the axis, a direction $d_{i}$ and a radius $r_{i}$.

The primitive shape model fitting error is computed using the $L^{2}$ distance. The selection of the right primitive model is based on the estimated cost of comparison of the plane, the sphere, and the cylinder. The minimum one is selected for the best fitting of the corresponding region.

$E_{\text {shape }}(j)=\left\{\begin{array}{l}E_{1} \propto \sum_{i} a\left(x_{i}\right)\left(n\left(x_{i}-\bar{x}\right)\right)^{2}, \\ \text { plane fitting } \\ \left.E_{2} \propto \sum_{i} a\left(x_{i}\right)\left(\left\|x_{i}-c_{i}\right\|_{2}-r_{i}\right)\right)^{2}, \\ \text { sphere fitting } \\ \left.E_{3} \propto \sum_{i} a\left(x_{i}\right)\left(\left\|\left(x_{i}-c_{i}\right) \times n\right\|_{2}-r_{i}\right)\right)^{2}, \\ \text { cylinder fitting }\end{array}\right.$

where $j$ is the number of models, $x_{i}$ is a vertex and $a\left(x_{i}\right)$ is the restricted Voronoi area of $x_{i}$. If a non-integer error estimation is used, $a\left(x_{i}\right)$ is often set to 1 for all the vertices. For the plane fitting, we follow the method using principal component analysis [13]. For sphere fitting, the cost function is nonlinear and the possible solution of Gauss-Newton method is prohibitive due to the computational cost, we follow a computing method of algebraic distance [21], [18] using the least squares method. For cylinder fitting, the Gaussian image of estimated surface normals [26] has been used to find the direction of the cylinder axis. The direction of such an axis is determined as the normal of a plane passing through the origin such that the sum of squared distances to the points in the Gaussian sphere is minimized.

Based on a hierarchical triangle face merging strategy [12], [13], each primitive shape model corresponding fitting parameters are computed and the approximation cost is estimated. The cost of hierarchically merging a set of triangles into a single representative cluster is the minimum of the approximation errors computed against the primitive

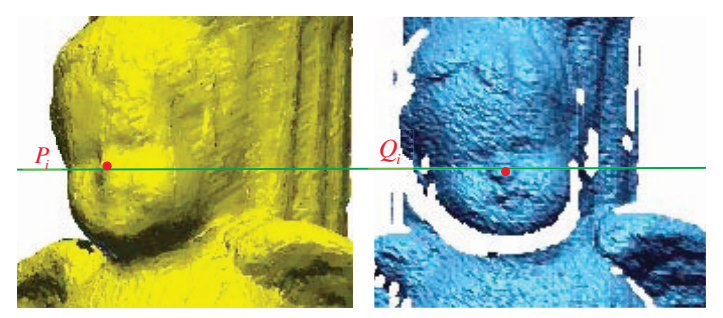

Figure 5. $a \mid b$ (a) A Low-resolution surface and a feature position. (b) A high-resolution surface patch and a related feature position.

models. This approach can be performed with some given numbers of segmentation regions (or parts) for a 3D shape.

The segmentation method is effective for surface registration on large and complex 3D shapes using hybrid scanning data. The method of hierarchical segmentation method does not require any seed information as initial starting point. Also, this approach is very efficient and fast for segmenting such large and complex 3D shape models even with unknown stronger scanning noises. Two examples of the results produced by this algorithm are shown in Fig. 4. A lowresolution global shape model with the size of $65.5 \mathrm{MB}$ is loaded for testing the algorithm. The partition of the surface shown in (a) has 178,951 clusters. The partition in (b) contains only 100 clusters. Note that the clusters are growing along the leg, the tail, the body and the foot on the 3D shape model of an anteater skeleton. Also note how the hierarchically clustered regions grow naturally into some semantic meaningful regions.

\section{Workflow and Implementation.}

\subsection{Original Raw Data Based Surface Registration}

Through our experiments, the noise distribution of 3D scanning data is not additive Gaussian noise but rather a more complicated nonlinear distribution including the influences of CCD camera, laser beams and object movements. The pre-processes of smoothing and denoising for local surfaces will heavily influence the accuracy of feature extraction and surface registration. In contrast to previous work [14], we extract local feature descriptors using the original raw data and smooth the registered surfaces in the final step.

To achieve a non-rigid global shape-driven surface alignment, we first propose to use the feature positions from the low-resolution scanned global shape model for adjusting the position and orientation of local patches. Some distinct geometric features [25], [14] on the low-resolution global shape model can be considered as the global features for local surface alignment in the presence of noises including occlusions, holes, distortions, e.g., shown in Fig. 5. Based on the global feature positions using the low-resolution global shape model, shown in the center of Fig. 3, we use a ThinPlate-Spline (TPS) regularization function [5] for aligning local surface patches to the low-resolution global shape 


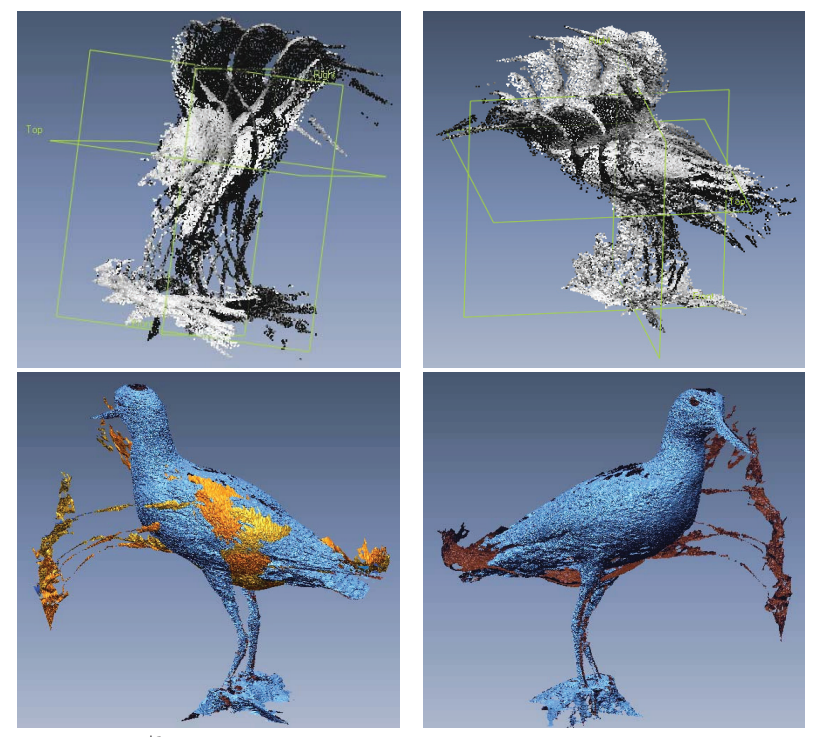

Figure 6. $\frac{a \mid b}{c \mid d}$ Surface registration error using only local surface patches even with some human supervision. (a)(b) Local surface patches. (c)(d) Registration results.

model and then improve the surface resolution using registered local surface patches.

\subsection{Local and Global Surface Registration}

When dealing with hybrid scanning data sets consisting of hundreds of high-resolution local surface scans, it is not practical to perform supervised pairwise registrations aligning each scan with other partially overlapping scans.

Using hybrid data, a low-resolution global shape model supplies a suitable initial alignment in two steps. (1) One could manually specify for each patch three points and their corresponding points on the global model, e.g., shown in Fig. 5. (2) The ICP algorithm refines this placement of the patches using extracted feature points of the patches and the global model. Then the patches are registered incrementally to each other in a growing process. After each such registration the merged patches are registered with the global model in a non-rigid way. In case of an incorrect merging of a patch the registration of the merged patch with the global model yields a poor quality. This can be detected and interactively corrected. For example, the large alignment errors in Fig. 6 can be corrected using global feature positions.

Furthermore, we consider several implementation aspects, including the search for closest points, choice of parameters, and efficiency. The efficiency of our approach depends on several factors. For searching nearest neighbors we apply k-d trees for speed up the searching [24], [28]. Parameters must be chosen that govern when a registration is considered satisfactory. In our case, the regularization parameter $\lambda$ during the global nonrigid-shape alignment is small, e.g., $\lambda=10^{-9 \sim-10}$. The non-rigid global surface alignment and refinement is adjusted with weakly bending energy following global shape features.

\section{Experimental Results and Discussions}

Experiments are carried out to evaluate and demonstrate the effectiveness of the suggested hybrid scanning system in terms of acquiring local detail scans and estimating uncertainty and confidence in various regions of the scanned objects with different genus complexities.

\subsection{Hybrid Scanning System and Data}

We use a stationary scanner and a hand-held laser scanner to construct such a hybrid scanning system. The stationary laser scanner on the tripod is a Minolta VIVID-900 scanner with an accuracy $0.16-0.22 \mathrm{~mm}$ which can scan object size between 0.1-1 $\mathrm{m}$ within a distance of 0.6-2.5 m. To optimize the scanning results, the right lens has to be chosen based on the distance between the object and the laser scanner, and the size of the object. The hand-held laser scanner is a "Polhemus FastSCAN" with an accuracy 0.75-1 mm which is a on-line 3D generating scanner via a transmitter and a receiver, connected to a processing unit in an magnetic field. It has a relatively low-resolution but provides a scanned global shape model in one scanning process.

To test the efficiency of the hybrid laser scanner system, several 3D shape models of different size, surface material, geometric complexity, and geometric structure were chosen in order to validate the efficiency and accuracy of the proposed approach. Fig. 2 shows a skeleton of an anteater (approximately $0.80 \mathrm{~m}$ long). For such complex objects a large number of high-resolution patches are required, in this case 370 scans. One such high-resolution patch is shown in Fig. 2 (c), and a low-resolution global shape model is shown in Fig. 2 (a) and (b). Fig. 11 shows the final recovered 3D model using hybrid scanned range images based on our suggested approach. The recovered anteater model is reconstructed based on aligning 370 high-resolution local surface patches to a segmented low-resolution global shape shown in Fig. 10. Total size is $1.162 \mathrm{~GB}$ with 30 millions vertices. The size of the final result in the Fig. 11 is 293 MB with 5.2 millions vertices after some down-sampling and thinning processes.

\subsection{Comparison of Methods}

The traditional approach for surface reconstruction from laser scans is to register and align local patches of the same (fine) resolution. Features for registration can be selected manually when the automatic feature extraction and surface alignment fails. Our approach is based on the use of hybrid scanning data, i.e., the high-resolution local surface patches are not only matched each other but also are registered on the low-resolution global shape model. All scanned local patches are aligned to the global shape model. In our exper- 


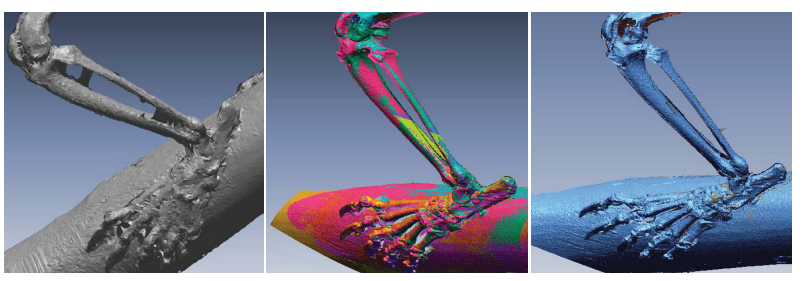

Figure 7. $a|b| c$. Registration of high-resolution local surface patches to low-resolution global shape model. (a) Low-resolution 3D shape model from the hand-held scanner. (b)(c) Registration results using high-resolution 3D surface patches together with the low-resolution 3D shape model (a).
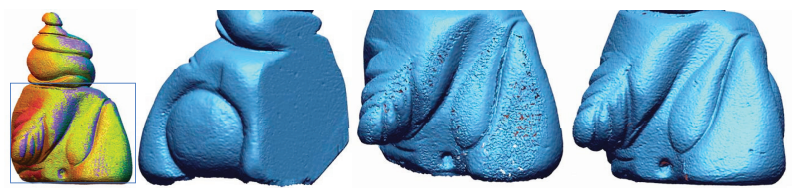

Figure 8. $a|b| c \mid d$. (a) A 3D shape model. When registering highresolution patches one side of the object registers well (b), while the other side has large distortions (c). Registration with hybrid data produces a better result (d).

iments the original raw scan data have been used, without any pre-filtering for noise reduction and smoothing.

We show some improved 3D surface registration results using hybrid scanning data, shown in Fig. 7. Fig. 7 (a) shows a section of the low-resolution complete shape model of the anteater, (b) shows the improved shape model using several high-resolution local surface patches, aligned on this low-resolution shape model. (c) shows the improved shape model in one color. Fig. 9 compares results of the classic and the hybrid registration approaches. Fig. 9 (b)(c)(d), obtained by registering only high-resolution patches shows gaps and distortions in the model. Since the surface registration starts from one side, accumulated global distortion appears at the other side. Fig. 9 (f-h) shows the results using the hybrid method. The local surface patches fit together. Although, some small distortions still appear due to some gaps in the low-resolution scanning data, shown in Fig. 9 (e), the resulting model is very close to the original object. In Fig. 8, we can see the similar performance. Overall, our approach provides information for global optimization, reduces the number of required scanning patches, easily fills holes, eliminates accumulated alignment errors, efficient computation and performance using segmented subshapes, and is robust to noise and outliers.

\section{Conclusions and Future Work}

We have presented an efficient approach for complex 3D shape acquisition and high-quality surface registration combining hierarchical shape approximation and hybrid geometric shape features. The method makes use of a natural global shape coverage for registering numerous highresolution local surface patches. To avoid the memory bot-

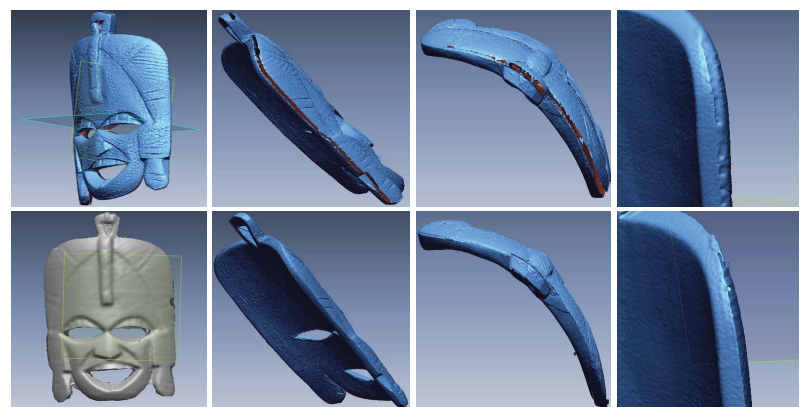

Figure 9. $\frac{a|b| c \mid d}{e|f| g \mid h}$. Top row shows registration using only highresolution patches: a high-resolution patch (a) and resulting errors (brown colored holes) (b-d). Bottom row shows registration with the hybrid method: the low-resolution global shape model (a) and high-quality registration using hybrid scanning data $(\mathrm{f}-\mathrm{h})$.

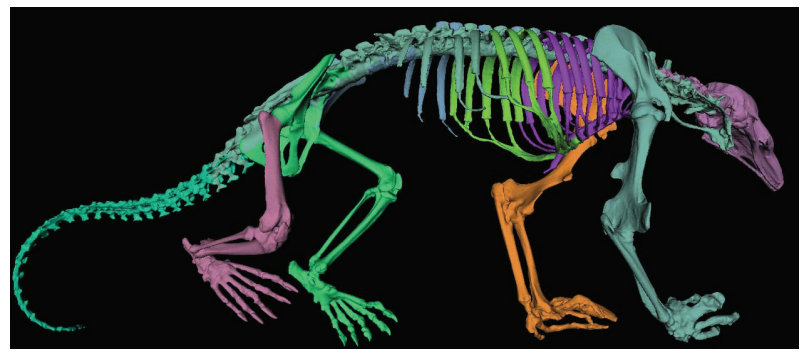

Figure 10. Semantic meaningful segmentation of complex 3D shape models using the proposed segmentation approach.

tlenecks, we proposed to segment the global shape model into sub-shapes which can achieve efficient local surface registration using the same global shape prior. The approach is robust to outliers of scans and can deal with some reflections, missing object parts, and may compensate for occlusion of objects in large high-resolution scans. Our experiments show that the joint utilization of global and local geometric shape information, together with an efficient segmentation and registration method, makes it reliable and efficient to reconstruct complex 3D shapes. The proposed approach can be applied for more practical 3D shape acquisition and surface registration applications.

\section{Acknowledgments}

The work was supported by the DFG GK 1042 "Explorative Analysis and Visualization of Large Information Spaces" at Universität Konstanz. The authors would like to thank anonymous reviewers for their useful comments.

\section{References}

[1] P. Alliez, D. Cohen-Steiner, O. Devillers, B. Levy, and M. Desbrun. Anisotropic polygonal remeshing. In Proc. ACM SIGGRAPH, pages 485-493, 2003.

[2] R. Benjemaa and F. Schmitt. A solution for the registration of multiple 3D point sets using unit quaternions. In $E C C V$, 1998. 


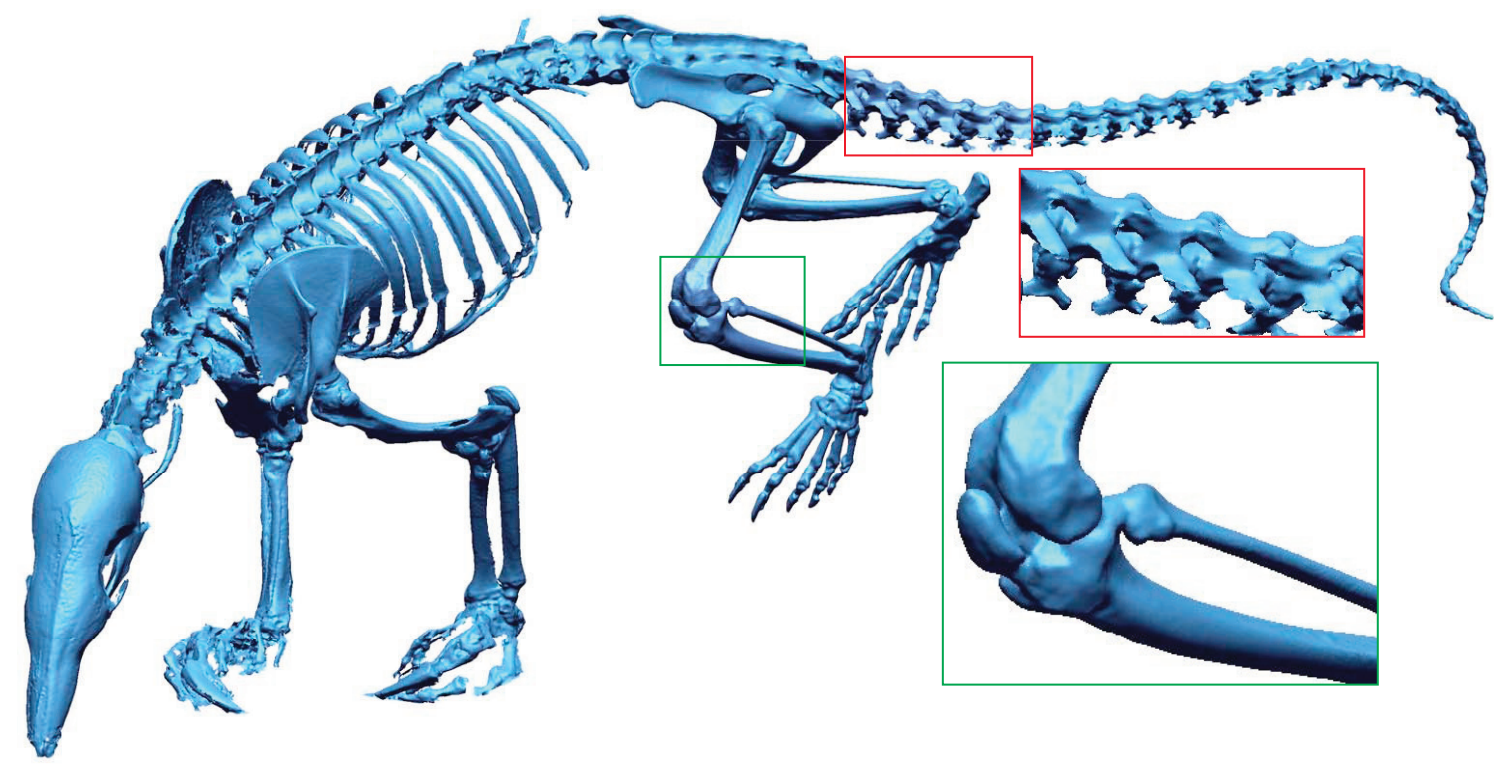

Figure 11. Acquired 3D shape model for an anteater skeleton using the hybrid scanning data. Size $=293 \mathrm{MB}, 5.2$ millions vertices.

[3] R. Bergevin, M. Soucy, H. Gagnon, and D. Laurendeau. Towards a general multi-view registration technique. IEEE Trans. on PAMI, 18(5):540-547, 1996.

[4] P. Besl and N. McKay. A method for registration of 3D shapes. IEEE Trans. on PAMI, 14(2):239-256, 1992.

[5] F. L. Bookstein. Principal warps: Thin-plate splines and the decomposition of deformations. IEEE Trans. on PAMI, 11(6):567-585, 1989.

[6] M. Botsch and O. Sorkine. On linear variational surface deformation methods. IEEE Transactions on Visualization and Computer Graphics, 14(1):213-230, 2008.

[7] B. Brown and S. Rusinkiewicz. Global non-rigid alignment of 3-D scans. ACM SIGGRAPH, 26(3), 2007.

[8] Y. Chen and G. Medioni. RANSAC-based DARCES: A new approach to fast automatic registration of partially overlapping range images. IEEE on PAMI, pages 1229-1234, 1999.

[9] H. Chui and A. Rangarajan. A new algorithm for non-rigid point matching. In CVPR, pages 44-51, 2000.

[10] D. Cohen-Steiner, P. Alliez, and M. Desbrun. Variational shape approximation. In ACM SIGGRAPH, pages 905-914, 2004.

[11] J. Duchon. Splines minimizing rotation-invariant seminorms in sobolev spaces. In Constructive Theory of Functions of Several Variables, pages 85-100. Springer-Verlag, 1977.

[12] M. Garland and P. Heckbert. Surface simplification using quadric error metrics. In ACM SIGGRAPH, pages 209-216, 1997.

[13] M. Garland, A. Willmott, and P. Heckbert. Hierarchical face clustering on polygonal surfaces. In ACM SIGGRAPH, pages 49-58, 2001.

[14] N. Gelfand, L. J. Guibas, L. Guibas, and H. Pottmann. Robust global registration. In Eurographics Symposium on Geometry Processing, 2005.

[15] N. Gelfand, L. Ikemoto, S. Rusinkiewics, and M. Levoy. Geometrically stable sampling for the ICP algorithm. In 3DIM, 2003.
[16] D. Hähnel, S. Thrun, and W. Burgard. An extension of the ICP algorithm for modeling nonrigid objects with mobile robots. IJCAI, 2003.

[17] M. Levoy, K. Pulli, B. Curless, S. Rusinkiewicz, D. Koller, and etc. The digital Michelangelo project: 3D scanning of large statues. SIGGRAPH, pages 24-28, 2000.

[18] A. Marco, F. Bianca, and S. Michela. Hierarchical mesh segmentation based on fitting primitives. The Visual Computer, 22(3):181-193, 2006.

[19] X. Pennec and J.-P. Thirion. A framework for uncertainty and validation of $3 \mathrm{D}$ registration methods based on points and frames. IJCV, 25:203-229, 1997.

[20] H. Pottmann, Q.-X. HuangG, Y.-L. Yang, and S.-M. Hu. Geometry and convergence analysis of algorithms for registration of 3D shapes. IJCV, 67(3):277-296, 2006.

[21] V. Pratt. Direct least-squares fitting of algebraic surfaces. ACM SIGGRAPHF, pages 145-152, 1987.

[22] K. Pulli. Multiview registration for large data sets. In 3DIM.

[23] L. Silva, O. Bellon, and K. Boyer. Robust Range Image Registration Using Genetic Algorithms and the Surface Interpenetration Measure, volume 60. World Scientific, 2005.

[24] R. Szeliski and S. Lavallee. Matching 3-D anatomical surfaces with non-rigid deformations using octree-splines. IJCV, 18(2):171-186, 1996.

[25] G. Taubin. Estimation of planar curves, surfaces, and nonplanar space curves defined by implicit equations with applications to edge and range image segmentation. IEEE Trans. PAMI, 13:1115-1138, 1991.

[26] T. Várady, G. Kós, and P. Benkö. Reverse engineering regular objects: simple segmentation and surface fitting procedures. In International Journal of Shape Modeling, volume 4, pages 127-142, 1998.

[27] G. Wahba. Spline Models for Observational Data, volume 59. SIAM, 1990.

[28] Z. Zhang. Iterative point matching for registration of free form curves and surfaces. IJCV, 13(2):119-152, 1994. 